\title{
Dysphagia in Perry Syndrome: Pharyngeal Pressure in Two Cases
}

\author{
George Umemoto $^{\mathrm{a}}$ Yoshio Tsuboi $^{\mathrm{b}}$ Hirokazu Furuya $^{\mathrm{c}}$ \\ Takayasu Mishima $^{b}$ Shinsuke Fujioka ${ }^{b}$ Naoki Fujii $^{d}$ Hajime Arahata ${ }^{d}$ \\ Miwa Sugahara $^{\mathrm{d}}$ Mitsuaki Sakai $^{\mathrm{e}}$ \\ ${ }^{a}$ Department of Oral and Maxillofacial Surgery, Faculty of Medicine, Fukuoka University, \\ Fukuoka, Japan; ${ }^{b}$ Department of Neurology, Faculty of Medicine, Fukuoka University, \\ Fukuoka, Japan; 'Department of Neurology, Kochi Medical School, Kochi University, \\ Kochi, Japan; ${ }^{d}$ Department of Neurology, National Hospital Organization Omuta National \\ Hospital, Fukuoka, Japan; ${ }^{\mathrm{e}}$ Department of Rehabilitation, National Hospital Organization \\ Omuta National Hospital, Fukuoka, Japan
}

\section{Keywords}

Perry syndrome $\cdot$ Dysphagia $\cdot$ Videofluorography $\cdot$ Manometry $\cdot$ Tongue pressure

\begin{abstract}
Background: To investigate the impact of dysphagia in Perry syndrome (PS), an autosomal dominant parkinsonism caused by mutation of DCTN1, which is associated with hypoventilation, depression, and weight loss. Case Presentation: We used tongue pressure measurements and manofluorography to investigate swallowing function in 2 patients with PS. Case 1 , a 60-year-old male showing parkinsonism, and case 2, a 49-year-old male admitted with pneumonia, were diagnosed as having PS based on the DCTN1 gene analysis. Case 1 showed a pharyngeal retention of the bolus on videofluorography (VF) and a few swallows were required for its passage into the esophagus. However, tongue pressure and manometry were within the normal range. This patient could eat a normal diet under supervision. Case 2 required artificial ventilation and tube feeding on admission. The VF image showed a slow transfer of the bolus, delayed swallow reflex, and pharyngeal retention of the bolus that required several swallows for its passage into the esophagus. The tongue pressure was within the normal range, but manometry showed a significant decrease in pressure at the hypopharynx and upper esophageal sphincter. The oral intake of the patients was limited to 2
\end{abstract}

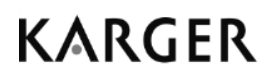

George Umemoto, DDS, PhD Department of Oral and Maxillofacial Surgery

Faculty of Medicine, Fukuoka University

7-45-1 Nanakuma, Jonan-ku, Fukuoka 814-0180 (Japan)

E-Mail george@minf.med.fukuoka-u.ac.jp 
cups of jelly per day. Conclusions: The investigation of swallowing dysfunction of 2 cases of PS showed that maintaining pharyngeal pressure within the normal range was very important for oral feeding success and prognosis.

\section{Background}

Perry syndrome (PS) (MIM 168605) is an autosomal dominant parkinsonian disorder accompanied by hypoventilation, depression, and weight loss $[1,2]$. This disorder is caused by mutation in the DCTN1 gene (MIM 601143), which is also the susceptibility gene for amyotrophic lateral sclerosis (ALS1, MIM 105400) [3]. PS is rapidly progressive and leads to death a few years after the symptomatic disease onset because of respiratory insufficiency. The age of onset ranges from 30 to 56 years with a mean age of $46 \pm 6.6$ years, and most patients present with progressive bradykinesia, or a depressive or apathetic mood that is often accompanied by weight loss [1,2]. Parkinsonism is usually mild with bradykinesia, rigidity, resting and postural tremor, and axial signs. Response to levodopa is not generally sustained, although it has been reported that some patients responded to high-dose levodopa. Motor impairment may be severe and the cachectic syndrome may be worsened by swallowing difficulties. Hypoventilation seen in the late stage of the illness, is a critical symptom leading to respiratory insufficiency and ultimately to death $[1,2]$. Therefore, among the parkinsonian diseases, patients with PS have a relatively poor prognosis. To date, there have been no reports on the pathomechanism of dysphagia in PS patients. In this study, we assessed swallowing function in 2 PS patients, using manometry and videofluorography (VF) concurrently to clarify the mechanisms underlying pharyngeal dysphagia.

\section{Case Presentation}

Case 1

A 60-year-old male was referred to the Omuta National Hospital because of parkinsonism. He had had a 5-year history of depression. At the age of 58 years, he had been diagnosed as having a familial Parkinson disease (PD), based on his bradykinesia, resting tremor in the upper extremities, small stepped gait, and impaired postural reflex, as well as family history of parkinsonism. He had also developed a mask-like face, rigidity in the neck and limbs, stooped posture, and a tendency to lean backwards. He was found to carry the p.F52L mutation in the DCTN1 gene (c.156T>G), and he was diagnosed as having PS (Fig. 1a) [4]. His maternal aunt was admitted to hospital at 48 years of age because of parkinsonism associated with hypoventilation and dysphagia. She was not able to communicate verbally because of severe cognitive impairment and has been tube fed.

At the age of 63 years, he suffered from frequent falls and difficulty in breathing while walking. He was admitted to Omuta National Hospital for further evaluation of the disease.

The VF image obtained at the age of 63 years indicated pharyngeal retention of the bolus, and a few swallows were required for its passage into the esophagus. There was no penetration or aspiration. The tongue pressure was measured using a handy probe (JM-TPM; JMS, Tokyo, Japan) and the obtained value of $29.3 \mathrm{kPa}$ was within the normal range [5]. Manofluorography was performed with VF, and manometry using a four-channel solid-state catheter placed transnasally (UniTip Catheter; Unisensor AG, Attikon, Switzerland) did not show any decrease in pressure at the hypopharynx or upper esophageal sphincter (UES) 
while swallowing of a slice of jelly (Fig. 2a). The values of maximum pressure at the hypopharynx and UES while swallowing a jelly were 802.5 and $303 \mathrm{~mm} \mathrm{Hg}$, respectively. Both the duration of pharyngeal contractions and the intervals from the beginning of the pharyngeal contraction to the end of UES relaxation were approximately $0.5 \mathrm{~s}$ and not prolonged. The patient was able to eat a normal diet, under the guidance of a speech therapist to minimize the risk of aspiration.

\section{Case 2}

A 49-year-old male was admitted to the Fukuoka University Hospital due to aspiration pneumonia. At the age of 46 years, he had developed depression. At the age of 48 years, his movements had slowed and he suffered from severe weight loss. At that time, he was treated with levodopa with little benefit. He was found to carry a p.G71A mutation in the DCTN1 gene (c. $212 \mathrm{G}>\mathrm{C}$ ), he was diagnosed as having PS. His father and elder brother were also diagnosed with the disease (Fig. 1b) [6]. His father developed parkinsonism and suddenly suffocated at 47 years of age. His eldest brother showed symptoms of parkinsonism at the age of 41 years that was accompanied by hypoventilation and dysphagia. Another brother died suddenly at 50 years of age.

At the age of 49 years, his daily activities deteriorated and he had difficulty in walking. During the admission, he underwent tracheostomy, and started to be maintained under artificial ventilation. Although he received nutritional support through a nasal feeding tube, 5 months after admission, gastrostomy was performed because of a lack of improvement in the swallowing function. Seven months after admission, he expressed a wish to eat orally; therefore, we performed an initial assessment of swallowing function at the time.

Initial VF performed at the age of 50 years showed slow transfer of the bolus and a delayed swallow reflex. Pharyngeal retention of the bolus was observed, and several swallows were required for its passage into the esophagus. But there was no penetration or aspiration because of the $30^{\circ}$ reclining position. The third VF examination at the age of 51 years revealed nasal reflux and penetration on swallowing $3 \mathrm{~mL}$ of liquid. The tongue pressure $(30.8$ $\mathrm{kPa}$ ) was within the normal range [5]; however, manometry showed a significant decrease in pressure at the hypopharynx and UES while swallowing a slice of jelly (Fig. $2 \mathrm{~b}$ ). The maximum pressures at the hypopharynx and UES while swallowing a jelly were 157 and $59.5 \mathrm{~mm}$ $\mathrm{Hg}$, respectively. However, the durations of pharyngeal contractions and interval from the beginning of pharyngeal contraction to the end of UES relaxation were not obviously prolonged, at approximately 0.6 and $0.9 \mathrm{~s}$, respectively. The patient is able to eat 2 cups of jelly per day by mouth and has not suffered from pneumonia since admission.

Their oral phase scores based on VF dysphasia scale [7] were approximately close to the mean value of the patients with advanced idiopathic PD, Hoehn and Yahr stage 4 and 5 [8], while their pharyngeal phase scores were equivalent to the worst level among the advanced PD patients. The study was approved by the Ethics Committee of the Fukuoka University Hospital 12-7-10(12-063). Informed consent about this study was obtained from the 2 participants.

\section{Discussion}

This is the first report to evaluate dysphagia in PS patients. Patients with idiopathic PD typically show reduced tongue pressure as dysphagia progresses $[8,9]$. Both of the PS patients presented in this study showed pharyngeal retention of the bolus on VF, and several 
swallows were required for the passage of the residue into the esophagus. However, the tongue pressure was within the normal range. On manometry while swallowing jelly, no drop in pressure was observed in case 1, whereas in case 2 there was a significant decrease in pressure at the hypopharynx and UES. Comparing the 2 current PS patients with patients with PD who had the same Hoehn and Yahr stage, the PS cases required more assistance with daily activities. This suggests that systemic diffuse atrophy could explain the dysphagia in the PS patients. However, tongue pressure was normal, even in case 2. Therefore, even if atrophy does occur in swallowing-related muscles, it may be localized to several specific muscle groups in PS patients.

In contrast to the findings in idiopathic PD [10], the pharyngeal retention on VF and decreased pharyngeal pressure but normal duration of the swallowing reflex on manometry in the 2 PS patients suggest that muscle weakness, rather than impaired coordination of swallowing movements, causes difficulty in passing the bolus through the pharynx. This hypothesis is consistent with the fact that aspiration/suffocation is common in PS patients $[1,2]$. However, it is difficult to generalize swallowing dysfunction in PS patients based on findings of only these 2 cases. Further studies are required to confirm the hypothesis and clarify the differences in the characteristics of dysphagia between idiopathic PD and PS. Our results highlight the need for medical staff to be alert not only to respiratory failure but also to suffocation or aspiration pneumonia secondary to pharyngeal weakness.

\section{Conclusion}

The result of our study shows that the symptom of swallowing difficulty in PS patients may be caused not by weakness of the tongue and/or impaired coordination of swallowing movements, but by specific pharyngeal muscle weakness. Manofluorography is a valuable method for the evaluation of pharyngeal dysphagia and for the guidance of swallowing by speech therapists to prevent suffocation in PS patients.

\section{Acknowledgments}

We are grateful for the support of this work by the Japan Society for the Promotion of Science and Grants-in-Aid for Scientific Research (C25463276 and B26860678).

\section{Statement of Ethics}

The study was approved by the Ethics Committee of the Fukuoka University Hospital 12-7-10(12-063). Written informed consent was obtained from the 2 participants for publication of this case report and the accompanying images.

\section{Disclosure Statement}

All authors have no competing interest. 


\section{Author Contributions}

G.U., H.A., M. Sugahara, and M. Sakai collected data and performed statistical analyses. G.U. wrote the manuscript and conceived the research hypothesis. Y.T., H.F., T.M., S.F., and N.F. reviewed and edited the manuscript and assisted in patient recruitment. All authors read and approved the final manuscript.

\section{References}

1 Wider C, Wszolek ZK: Rapidly progressive familial parkinsonism with central hypoventilation, depression and weight loss (Perry syndrome) - A literature review. Parkinsonism Relat Disord 2008;14:1-7.

2 Tsuboi Y, Wszolek ZK, Kusuhara T, Doh-ura K, Yamada T: Japanese family with parkinsonism, depression, weight loss, and central hypoventilation. Neurology 2002;58:1025-1030.

-3 Vilarino-Guell C, Wider C, Soto-Ortolaza AI, Cobb SA, Kachergus JM, Keeling BH, Dachsel JC, Hulihan MM, Dickson DW, Wszolek ZK, Uitti RJ, Graff-Radford NR, Boeve BF, Josephs KA, Millar B, Boylan KB, Gwinn K, Adler CH, Aasly JO, Hentati F, Destee A, Krygowska-Wajs A, Chartier-Harlin MC, Ross OA, Rademakers R, Farrer MJ: Characterization of DCTN1 genetic variability in neurodegeneration. Neurology 2009;72:2024-2028.

-4 Mishima T, Ishikawa T, Imamura K, Kondo T, Koshiba Y, Takahashi R, Takahashi J, Watanabe A, Fujii N, Tsuboi Y, Inoue H: Cytoplasmic aggregates of dynactin in iPSC-derived tyrosine hydroxylase-positive neurons from a patient with Perry syndrome. Parkinsonism Relat Disord 2016;30:67-72.

-5 Utanohara Y, Hayashi R, Yoshikawa M, Yoshida M, Tsuga K, Akagawa Y: Standard values of maximum tongue pressure taken using newly developed disposable tongue pressure measurement device. Dysphagia 2008;23:286-290.

-6 Mishima T, Fujioka S, Kurisaki R, Yanamoto S, Higuchi MA, Tsugawa J, Fukae J, Neshige R, Tsuboi Y: Impulse control disorders and punding in Perry syndrome. Parkinsonism Relat Disord 2015;21:13811382.

7 Han TR, Paik NJ, Park JW, Kwon BS: The prediction of persistent dysphagia beyond six months after stroke. Dysphagia 2008;23:59-64.

-8 Umemoto G, Tsuboi Y, Kitashima A, Furuya H, Kikuta T: Impaired food transportation in Parkinson's disease related to lingual bradykinesia. Dysphagia 2011;26:250-255.

$\checkmark 9$ Sung HY, Kim JS, Lee KS, Kim YI, Song IU, Chung SW, Yang DW, Cho YK, Park JM, Lee IS, Kim SW, Chung IS, Choi MG: The prevalence and patterns of pharyngoesophageal dysmotility in patients with early stage Parkinson's disease. Mov Disord 2010;5:2361-2368.

10 Ertekin C, Tarlaci S, Aydogdu I, Kiylioglu N, Yuceyar N, Turman AB, Secil Y, Esmeli F:

Electrophysiological evaluation of pharyngeal phase of swallowing in patients with Parkinson's disease. Mov Disord 2002;17:942-949. 
a

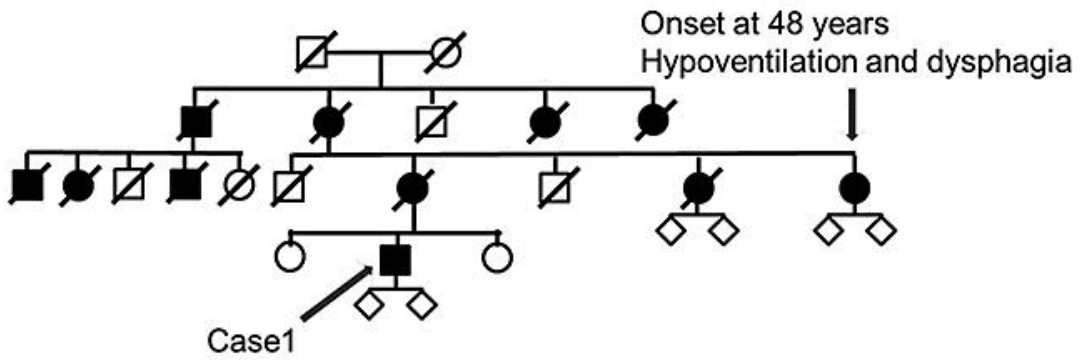

b

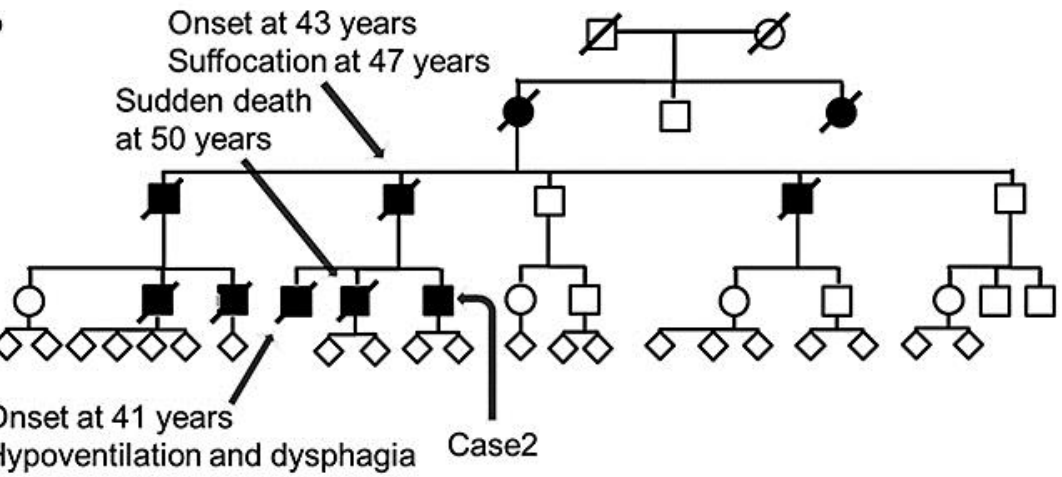

Fig. 1. Pedigrees of case 1 (a) [4] and case 2 (b) [6]. For the pedigrees (a, b), standard pedigree symbols were used. Round symbols indicate females, squares indicate males, and diagonal lines indicate that the individual is deceased. Black symbols indicate affected members. Diamonds were used to disguise gender. Family A was previously described in the paper reported by Mishima et al. [4], and family B was reported by Tsuboi et al. $[2,6]$. 
a, Case 1
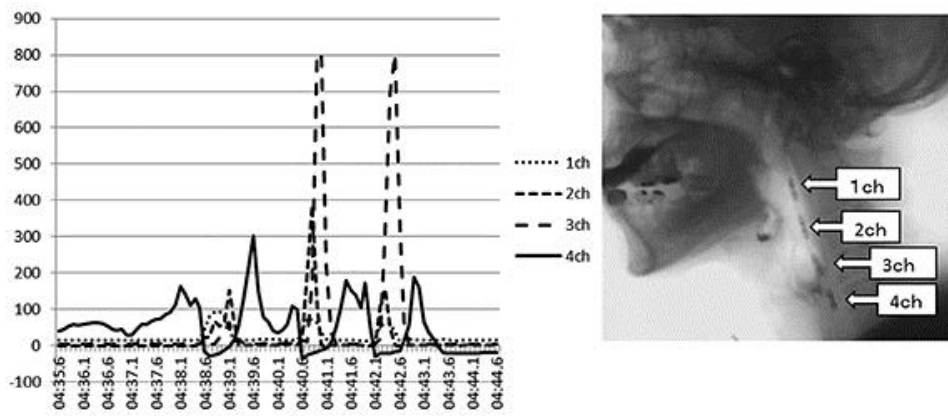

b, Case 2

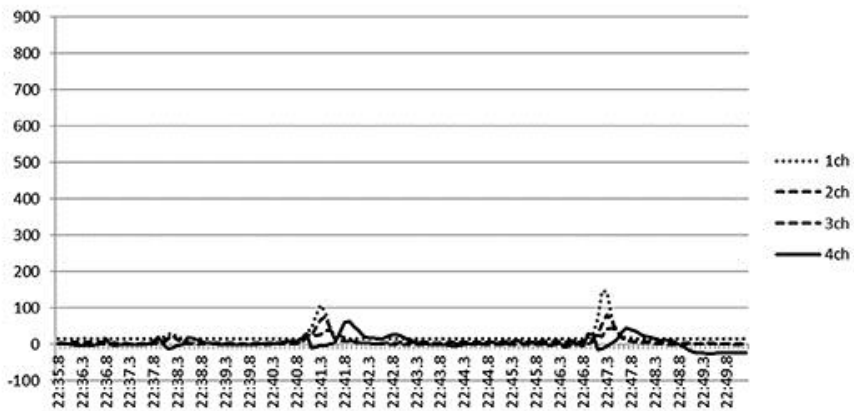

Fig. 2. Conventional line tracings on manofluorography in case 1 (a) and case 2 (b). Monofluorography performed in case 1 revealed a swallowing pressure maintained at a normal level, but in case 2, it showed a significant decrease in pressure at the hypopharynx and UES. UES, upper esophageal sphincter. 